\title{
Clinical impact of apparent structural and functional similarities between extracellular vesicles RNA (EVs RNA) and chronic RNA viruses
}

\author{
Wael Fouad Nassar ${ }^{1,2}$, Mostafa Abdel Aziz Mostafa ${ }^{2}$ \\ ${ }^{1}$ Department of nephrology, Sahel Teaching Hospital, General Organization of Teaching Hospitals and Institutes (GOTHI), Cairo. Egypt \\ ${ }^{2}$ Department of nephrology, kidney transplantation unit, October Six University Hospitals, Cairo. Egypt
}

\section{Email address:}

Hegaz_wn@yahoo.com (W. Nassar)

\section{To cite this article:}

Wael Fouad Nassar, Mostafa Abdel Aziz Mostafa. Clinical Impact of Apparent Structural and Functional Similarities between Extracellular Vesicles RNA (EVs RNA) and Chronic RNA Viruses. Cell Biology. Vol. 2, No. 5, 2014, pp. 41-48. doi: 10.11648/j.cb.20140205.11

\begin{abstract}
Disruption of nucleoprotein contents of cells can be considerd a reflection of cell stress phase rather than a reflection of the disease state of organs. Disruption of normal cellular protein machinery would occur as a result of cell exposure to stressors. Stressors can be extracellular or intracellular (e.g. infections, malnutrition, obesity, physical or mental exertion and other ecologic factors). Repeated tissue injury results in repeated altered remodeling of damaged tissues and may lead to tissue fibrosis and possibly organ failure (e.g. autoimmune or malignancies). Duration, severity and frequency of cell exposure to stressors have been proposed to exert negative impact on cells; possibly through the different types of extracellular vesicles (EVs) shed by cells involved in each exposure and consequently affect the mechanisms of cell function. These cumulative effects simulate, at least in part, the behavior of chronic RNA viruses (e.g. HCV, Herpes viruses and HIV) which may exert relatively harmless effect and remain dormant within the human body for months and years. We hypothesis that: EVs produced by different types of cells in response to exposure to stress, may behave like persistent self-induced particles as if they were auto-viruses and may need to be neutralized to abolish their proposed role in disease induction and/or progression, or otherwise they might provoke serious diseases.
\end{abstract}

Keywords: Extracellular Vesicles (EVs), Exosomes, Human Endogenous Retroviruses (HERVs), Horizontal Gene Transfer, Auto-Viruses

\section{Introduction}

Many diseases are described as resistant, recurrent and chronic. These terms though can be attributed to many factors; however, the net result is clearly; incomplete cure of patients. Since Valadi and co-workers elaborated the capability of circulating EVs to horizontally transfer functioning gene materials between cells [1], many researchers have been working to exploit the role EVs may play in the pathogenesis of different diseases as infections, malignancies and autoimmune diseases. Moreover, EVs released from different tumor cells, viruses and parasites may also kill immune cells at a distance to evade the immune system [2]. Recently, evidences are accumulating for the involvement of $\mathrm{T}$ cells in immune responses against human endogenous retroviruses (HERVs) in HIV-infected individuals [3].
EVs may influence the behavior of the recipient cells by different mechanisms. They may directly stimulate the cells by a surface interaction, they may act by transferring receptors proteins between cells, or may deliver proteins to target cells [23-25], and they may induce epigenetic changes in target cells by transferring genetic material $[1,26,27]$.

Some virus infections may affect their target organs through "latent mode". They may exist relatively harmless and remain dormant within the human body for months and years with disruption of healthy cell homeostasis resulting in disease (e.g. herpes virus) $[4,5]$. Moreover, some viruses can cause lifelong or "chronic infections", were they continue to replicate despite the host's defense mechanisms (e.g. HCV and HBV) [6,7].

Not only EVs released from infected cells can trigger 
immune reaction but also cell-derived vesicles from stressed cells can trigger the production of pro-inflammatory cytokines by activating targeted cells independent of the presence or absence of previous infections beside their ability to deliver gene materials to the target cells [8].

All these data led to consider the hypothesis of EVs produced by different types of cells in response to exposure to stress (infectious or non-infectious), may behave as if they were persistent self-induced particles or auto-viruses.

We'll be addressing some apparent structural and functional similarities between EVs and viruses emphasizing the role EVs might play in both viral infections and inflammation which could be supporting this hypothesis.

\subsection{Extracellular Vesicles (EVs)}

Extracellular Vesicles (EVs) or microvesicles and sometimes called microparticles or more accurately nanoparticles, is a term used for vesicles that are released from the plasma membrane under basal conditions or during cell stress [9]. Microvesicles are present in most, if not all, biological fluids, atherosclerotic plaques and conditioned culture medium $[10,11]$. Based on their biophysical properties and biogenesis, many types of vesicles have been described [12]. Herein, we'll be focusing on the two major groups of non-apoptotic vesicles that are defined by their biogenesis: microvesicles and exosomes.

Exosomes are smaller than microvesicles with size range between 30 and $120 \mathrm{~nm}$ and the floating density ranges between 1.13 and $1.19 \mathrm{~g} / \mathrm{ml}$ [21]. Exosomes are cell-derived vesicles that are present in almost all biological fluids, including urine, blood, ascites and cerebrospinal fluid [13]. Exosomes are described as homogenous vesicles formed of mRNAs, microRNAs (miRNA), proteins, and signaling molecules [21, 75] surrounded by a bi-lipid membrane enriched in lipid rafts of cholesterol, sphingomyelin, and ceramide [12]. Exosomes express cell type-specific proteins components which, presumably, reflect the biological function of the parent cell (e.g., dexosomes for dentritic cellderived exosomes are enriched with major histocompatibility complex (MHC) class I and II and express co-stimulatory molecules like CD54, CD80 and CD86 and also prostaspmes for prostate-derived exosomes) [7], exosomes from synovial fluid contain citrullinated proteins (eg. fibrin $\alpha$-chain fragment, fibrin $\beta$-chain, fibrinogen $\mathrm{D}$ fragment and $\mathrm{Sp} \alpha$ receptor) which might play an important role in converting nonimmunogenic proteins into autoimmunogenic proteins [7] Exosomes also express molecules that are considered specific markers of exosomes of different origin, such as tetraspanin family members (CD63, CD9, CD81, CD82), flotillin, Tsg101, Alix and other components of the endosomal sorting complex required for transport (ESCRT). Moreover, some exosomes may contain heat shock proteins (Hsp70, Hsp90) and Rab-GTPases [12, 14]. Expression of phosphatidylserine (PS) on exosomes membrane is still controversial and remains to be determined [15]. Exosomes are the only vesicles originating from inward budding of cell membranes (endocytic origin), these vesicles directly fuse with the plasma membrane and release exosomes into the extracellular space [16].

Microvesicles are generated by direct budding of the cell membrane, with a size ranging from 50 to $1000 \mathrm{~nm}$, express surface markers such as integrin- $\beta$, CD40 ligand and selectins such as plasma selectins and surface protein receptors that characterize the membrane composition of the cells of origin [17-20].

Because of their small size, vesicles are below the detection range of conventional detection methods. Vesicles are isolated by multiple sequential differential centrifugation (100000-200000) g. Besides, filtration can be applied to isolate vesicles. Although the pore size of filters is often well defined, increasing forces have to be applied with decreasing pore size, which may result in artifacts [7].

Distinction based on biogenesis and size or protein and lipid composition, between various types of vesicles, remains confusing because the markers used for defining vesicles are frequently not exclusive, and may vary depending on the cell of origin $[21,22]$. Besides, the size ranges of microvesicles and exosomes may overlap. For this reason, the generic term "extracellular vesicles (EV)" has been suggested for all secreted vesicles [14], and we'll be using the term EVs representing exosomes or microvesicles.

EVs may influence the behavior of the recipient cells in different mechanisms. They may directly stimulate the cells by a surface interaction, they may act by transferring receptors proteins between cells, or may deliver proteins (e.g. products of oncogenes or caspase- 1 protein) to target cells [23-25], and they may induce epigenetic changes in target cells by transferring genetic material $[1,26,27]$.

\section{Viruses}

Viruses are organic nano-structures which interact with living organisms. They do not have their own metabolism; so, require a host cell to make new products. They therefore cannot naturally reproduce outside a host cell [28]. Most studied viruses have a diameter between 20 and $300 \mathrm{~nm}$. Virion, a complete virus particle, consists of nucleic acid (DNA or RNA) surrounded by a protective coat of protein called a capsid, formed of identical protein subunits called capsomeres [29]. Enveloped viruses possess a lipid "envelope" derived from the host cell membranes [30, 31].

According to the type of viral nucleoprotein structure, virus is either DNA virus or RNA virus. The vast majority of viruses have RNA genomes. Irrespective of nucleic acid type the genome can be either single-stranded or double-stranded. The single strands are said to be either positive-sense or negativesense, depending on their polarity. Positive-sense viral RNA can be immediately translated by the host cell, whereas, negative-sense viral RNA must be converted to positive-sense RNA by an RNA polymerase enzyme before translation. However, several types of ssDNA and ssRNA viruses have genomes that are ambisense in that transcription can occur off both strands in a double-stranded replicative intermediate [32]. Viruses can undergo genetic change by several mechanisms; 
including "antigenic drift" where individual bases of the DNA or RNA mutate to other bases [33, 34] or "antigenic shift" where a major change, recombination or re-assortment, in the genome of the virus takes place [35]. This can occur when viruses infect cells simultaneously; it is common to both RNA and DNA viruses [36].

Because viruses are non-cellular structures they use the machinery and metabolism of host cell to produce multiple copies, and they assemble in the cell. Stages in the life cycle of viruses include attachment, penetration, un-coating, replication and release. The genome replication of most DNA viruses takes place in the cell's nucleus, while the genome replication of most RNA viruses usually takes place in the cytoplasm. Viruses must generate mRNAs by different mechanisms from their genomes to produce proteins and replicate themselves [37].

Cytopathic effect is the extensive range of structural and biochemical effects that viruses have on the host cell. Most virus infections eventually lead to cell death because of cell suppression by virus-specific proteins, interestingly, not all of which are components of the virus particle [38]. At the cellular level, different mechanisms by which viruses produce disease in multicellular organisms have been described, "lytic mode" with subsequent cell lysis and death and "latent mode" with disruption of healthy cell homeostasis resulting in disease, they may exist relatively harmless and remain dormant within the human body for months and years (e.g. Herpes virus) [39, 40]. Moreover, some viruses can cause lifelong or "chronic infections", where viruses continue to replicate despite the host's defense mechanisms (e.g. HCV and HBV) [41, 42].Virus-like particles (VLPs) resemble viruses, expressing viral structural proteins, such as envelope or capsid but are non-infectious because they do not contain any viral genetic material [43]. Similarities between EVs and viruses:

EVs and viruses show apparently structural similarity. EVs are formed of mRNAs, miRNAs, proteins, and signaling molecules contained in a lipid membrane from the host cell membrane, while viruses are formed of nucleic acid (DNA or RNA) surrounded by a protective coat of protein called a capsid. Enveloped viruses possess a lipid "envelope" derived from the host cell membranes [30, 31]. Although EVs structure does not obey the main morphological rules of viruses, however, it has been reported that some viruses have complex structures that are unrelated to any other forms of virus [44].

Virus attachment to the recipient cell is mediated through specific binding proteins of the viral capsid and specific receptors on the cell surface of the host, this "viral entry" is thought to be receptor mediated endocytosis or membrane fusion. Some viruses have evolved mechanisms that inject their genome into the host cell across the cell wall, while the viral capsid remains outside (un-coating) [45]. Similar mechanisms are nearly applied to EVs has been reported, where microvesicles released from a cell expresses adhesion molecules related to this cell and then they are captured by specific receptor-ligand interaction which specifically recognize them rather than just by any cell [14].

Soon after the virus entry to the infected cell, it replicates through synthesis of viral messenger RNA (mRNA) then viral genome replication mediated by regulatory protein expression [45]. Meanwhile, EVs contain selected patterns of mRNA and miRNA involved in the intracellular trafficking of RNA, suggesting a dynamic regulation of RNA compartmentalization in EVs [24]. Moreover, Components of RNA-induced silencing (siRNA) complexes are present in EVs, suggesting that this complex may be involved in the sorting of miRNAs within exosomes [46].

Following the structure-mediated self-assembly of the virus particles, some modification (sometimes called maturation) occurs after the virus has been released from the host cell (e.g, HIV) [45].Valadi and co-workers reported that many of the RNA contained in the EVs were not detected in the cytoplasm of their donor cells, raising the possibility of the presence of extracellular selective sorting mechanisms of the genetic proteins at the EVs level [1].

Some viruses undergo a lysogenic cycle where the genetic material is incorporated in the DNA of the gene substance of the infected cell chromosomes and the virus replicates only when the infected cell divides [47]. Independent of their characteristic structures or mechanism of biogenesis, extracellular vesicles (EVs) has been recently described as a new vehicle of horizontal transfer of genetic information between cells [48]. mRNA can be translated in the recipient cells, microRNAs are known to regulate more than $80 \%$ of all protein-encoding genes and the long non-coding RNA may be incorporated in epigenetic conformation and induce changes in the cell phenotype [24, 49, 50].

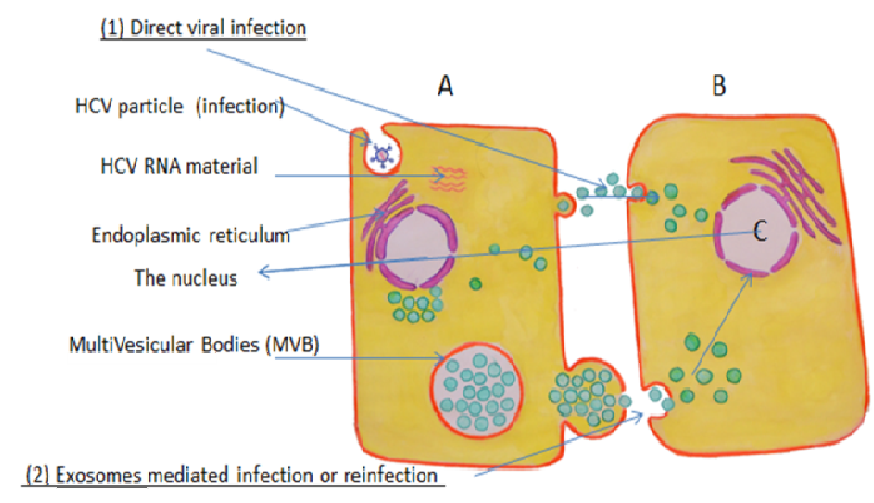

Figure (1). HCV infected cell may transmit the virus material to noninfected cell either by:

(1) Cell lysis and direct infection by the HCV nucleic substance.

(2) Cell re-infection by exosomes.

Exosomes from HCV-infected cells were capable of transmitting infection to naive cells and establishing a productive infection. Even with subgenomic replicons, lacking structural viral proteins, exosome-mediated transmission of HCV RNA was observed [54]. HCV entry receptors may partly contribute to exosome uptake even in the absence of viral envelope or core proteins [55]. The cytopathic effect of HCV infection differs between complete virus genome and exosome mediated hepatocyte infection (reinfection). While complete free virus genome mostly produces cell lysis and apoptosis, exosomes mediated infection or reinfection, can produce an antibodyresistant cell-to-cell transmission route and to establish a productive infection even in the absence of viral envelope or core proteins $[57,58,59]$. 


\subsection{Role of EVs in Viral Infections}

After the target cell being infected by the hepatitis $\mathrm{C}$ virus, (Figure 1), core proteins tend to promote cell death through up-regulation of caspases [51], Bax [52] and Fas-ligand [53], while the envelope proteins seem to favor cell growth, transformation and oncogenicity, the balance in-between these viral proteins seems to be crucial in defining the consequences of $\mathrm{HCV}$ infection [54]. This emphasizes that each part of the virus RNA may independently initiate a definite consequences of conformational gene modulations of the target cell [54]. Besides, HCV entry receptors may partly contribute to exosome uptake even in the absence of viral envelope or core proteins [55]. HCV entry receptors CD81 and SR-BI are also known to localize in lipid rafts of exosomes [56], supporting a hypothetical role of these receptors in exosome uptake by the neighboring healthy hepatocytes. The cytopathic effect of HCV infection differs between complete virus genome and exosome mediated hepatocyte infection (reinfection). While complete free virus genome mostly produces cell lysis and apoptosis, exosomes mediated infection or reinfection, can produce an antibodyresistant cell-to-cell transmission route and establish a productive infection even in the absence of viral envelope or core proteins [57, 58, 59]. Moreover, in membranoproliferative (mesangiocapillary) glomerulonephritis (MPGN) associates HCV, mesangial expansion is largely attributed to macromolecular deposits and to lesser extent the direct pathogenetic effect of the virus [60], supporting the hypothetical role of exosome in transmitting HCV genetic information to the kidney [61] even in the absence of viral envelope or core proteins [56].

Recently, it has been shown that exosomes released from HIV-infected cells contain negative regulatory factor, which induces apoptosis of uninfected cells though these cells do not contain the RNA of the virus itself [62], besides, EVs produced in response to HIV virus are more infectious to CD4 T cells than cell-free viral particles [63].

Moreover, human herpes virus 4 or EBV encodes RNAs that can be transferred from infected to uninfected cells by EVs [64-66]. The uptake of EVs from EBV-infected cells by epithelial cells results in activation of growth-stimulating signaling pathways and can manipulate the growth characteristics of neighboring cells $[67,68]$.

Viral particles or molecules associated with viral infection (not necessarily the whole genome) can be transmitted to adjacent uninfected cells via exosomes and become functional [69]. But the function of these particles or molecules released via the EVs is different from that of the virus itself. It has been postulated that pathogens, like viruses, have "hijacked" cell-derived vesicles as a transport vehicle to exchange genetic information between cells without the risk of being recognized by the immune system $[69,70]$.

Moreover, infected cells with hepatitis B virus, pox viruses, or herpes simplex virus, release EVs containing empty viral particles exposing viral glycoproteins in concentrations up to 10,000 fold higher than that of the infectious particles, suggesting that these vesicles may act as potential decoys to distract the immune system $[67,68]$.These terms (deceive, hijack, decoy, etc.), have used frequently to describe failure of the immune system to recognize infective agents. This is possibly might be because the genetic information which is carried by EVs belongs to the host and secreted as consequences of the viral infection but they are no longer carry the gene finger-print of the virus itself to be recognized by the immune system, arising the possibility that EVs RNA might be behaving as if they were like viruses with different mode of infectivity than that of the free virus itself [69].

Human endogenous retroviruses (HERVs) vesicles are virus genome that occupies as much as $4.9 \%$ of the human genome [70]. They are integrated into the human chromosome and are transmitted vertically as Mendelian genes. Although, human endogenous retrovirus particles (EVs) are released by some transformed cells, they have never been shown to be infectious [71]. Recently, evidences accumulating for involvement of $\mathrm{T}$ cells in immune responses against HERVs in HIV-infected individuals [3]. The hypothesis that HIV induces HERV expression in HIVinfected cells led to the proposal that a vaccine targeting HERV antigens could specifically eliminate HIV-infected cells. This observation supports the notion of synergism between EVs of old viral infections and newly developed once and raises the possibility of capturing or neutralizing these EVs as a new therapeutic modality.

\subsection{Role of EVs in Inflammation}

Cell response to stress, which may be physical, chemical or biological factor(s), is the ability of the cell to sense disturbance, and initiates a series of molecular reactions for maintenance, repair, adaptation, remodeling and survival. Demirovic and his colleague described seven main distinct pathways at the molecular level which are integral to the property of cell homeodynamics. These pathways are: antioxidant response, unfolded protein response (UPR), inflammatory response, heat shock response (HSR), sirtuin response, autophagic response and DNA damage response (DDR) [72]. Although it is unknown to what extent cellderived vesicles following cell exposure to stress contribute to the maintenance of cell homeostasis, it is tempting to speculate that vesicles have a protective role for cells from extracellular and intracellular stress [9].

Cell-derived vesicles from stressed cells can trigger the production of pro-inflammatory cytokines by activating targeted cells independent of the presence or absence of previous infections. This activation results in expression and production of tissue factors and interleukins [73]. The elegant experiment of Deng and his colleagues shows that EVs derived from adipose tissue when intravenously injected into wild-type C57BL/6 mice resulted in development of insulin resistance, whereas injection into TLR-4 knockout B6 mice the insulin resistance was not marked due to the effect of increased levels of TNF- $\alpha$ and IL-6 [7]. Despite the complexity and multifactorial nature of $\mathrm{T} 2 \mathrm{D}$, metabolic inflammation is the most common step leading to insulin 
resistance in the disease. Exosome-like vesicles released from adipose tissue are taken up by peripheral blood monocytes, which then differentiate into macrophages producing increased levels of TNF- $\alpha$ and interleukin-6 [74]. Based on this experiment, the gradual progressive dissemination of insulin resistance throughout the body may be attributed, at least in part, to EVs released from inflamed adipose tissues which may open the gate for a new modality of treatment for insulin resistance in diabetic patients [74].

Extracellular vesicles from human atherosclerotic plaques have been shown to mediate the functional transfer of ICAM-1 to endothelial cells of neighboring healthy cells, thereby promoting the adhesion of monocytes and transendothelial migration [70]. Thus, plaque EVs may further facilitate atherosclerotic plaque progression, simulating a slowly progressing viral infection.

Another example linking inflammatory processes to EVs shown in preeclampsia patient blood contains elevated levels of the placenta derived "syncytio-trophoblast EVs" that bind to the monocytes and endothelial cells thus triggering the production of pro-inflammatory cytokines and hence, it may contribute to the increased systemic inflammatory responsiveness in preeclampsia [72].

The ability of EVs to modulate the inflammatory response is not limited to blood. Autologous EVs from synovial fluid and EVs from T cells, monocytes, and platelets trigger the production and release of interleukins 6 and 8 , matrix metalloproteases, monocyte- chemotactic proteins 1 and 2, vascular endothelial growth factor (VEGF), and ICAM-1 by synovial fibroblasts, indicating that these vesicles may enhance the destructive activity of these fibroblasts in rheumatoid arthritis [71] in absence of true infection.

\section{Conclusion}

EVs RNA produced by autologous release from the mother cell in response to stress or produced by recurrent spontaneous unnoticed or improperly treated infections and their contribution to disease persistence or progression needs to be thoroughly investigated. Cell-derived EVs have the ability to transfer receptors and stimulate the production of inflammatory mediators. It has been recently reported that inappropriate clearance of apoptotic vesicles is considered to be the primary cause of developing systemic autoimmune diseases.

We hypothesis that: EVs produced by different types of cells in response to stress, may behave like persistent selfinduced particles as if they were auto-viruses and may need to be neutralized to abolish their proposed role in disease induction and/or progression, or otherwise they might provoke serious diseases.

According to this hypothesis, the to-date markers of inflammatory disorders are unable to identify the presence or absence of pathognomonic EVs and their role in persistence or progression of diseases. EVs may continue to disrupt the epigenetic products of the target organ(s) cells even after cessation of the stress stimuli and negatively affect the forthcoming stressful conditions particularly with the possibility of extracellular sorting mechanisms of EVs contents that may behave like persistent self-induced particles as if they were auto-viruses, as they simulate in part the latency mode of chronic viral infections.

One may expect that the hypothesis of auto-virus may improve the general health care outcome and open a new window for novel diagnostic and therapeutic modalities.

\section{Acknowledgments}

We would like to acknowledge Professor Doctor Rashad S. Barsoum, professor of internal medicine and nephrology, faculty of medicine, Cairo University, for his useful and helpful discussions and comments. We wish also to acknowledge Dr. Mayar WN, who put our imagination onto real illustration.

\section{References}

[1] Valadi, H.; Ekström, K.; Bossios, A.; Sjöstrand, M.; Lee, J.J.; Lötvall, J.O (2007) Exosome-mediated transfer of mRNAs and microRNAs is a novel mechanism of genetic exchange between cells. Nat. Cell Biol. 9, 654-659.

[2] Canchaya C, Fournous G, Chibani-Chennoufi S, Dillmann ML, Brüssow H (2003) Phage as agents of lateral gene transfer. Current Opinion in Microbiology.6 (4):417-24.

[3] Mahy WJ \& Van Regenmortel MHV (eds) (2009). Desk Encyclopedia of General Virology. Oxford: Academic Press; $\mathrm{p}$ 24 Whitley RJ, Roizman B (2001). Herpes simplex virus infections. Lancet.357 (9267):1513-8. Barton ES, White DW, Cathelyn JS, et al(2007).. Herpesvirus latency confers symbiotic protection from bacterial infection. Nature.447 (7142):326-9.

[4] Bertoletti A, Gehring A (2007). Immune response and tolerance during chronic hepatitis B virus infection. Hepatol. Res.37 Suppl 3:S331-8.

[5] Rodrigues C, Deshmukh M, Jacob T, Nukala R, Menon S, Mehta A (2001). Significance of HBV DNA by PCR over serological markers of HBV in acute and chronic patients. Indian journal of medical microbiology.19 (3):141-4.

[6] Wang JG, Williams JC, Davis BK, Jacobson K, Doerschuk CM, Ting JP, and Mackman N (2011). Monocytic microparticles activate endothelial cells in an IL1betadependent manner. Blood 118:2366-2374.

[7] Edwin van der Pol, Anita N. Bo“ing, Paul Harrison, Augueste Sturk, and Rienk (2012) Classification, Functions, and Clinical Relevance of Extracellular Vesicles. Nieuwland, Pharmacol Rev 64:676-705

[8] Berckmans RJ, Sturk A, van Tienen LM, Schaap MC, and Nieuwland R (2011). Cell-derived vesicles exposing coagulant tissue factor in saliva. Blood 117:3172-3180.

[9] Morel O, Jesel L, Freyssinet JM, and Toti F (2011) Cellular mechanisms underlying the formation of circulating microparticles. Arterioscler Thromb Vasc Biol 31:15-26. 
[10] Mathivanan S, Ji H, and Simpson RJ (2010) Exosomes: extracellular organelles important in intercellular communication. J Proteomics 73:1907-1920

[11] Vella LJ, Greenwood DL, Cappai R, Scheerlinck JP, and Hill AF (2008) Enrichment of prion protein in exosomes derived from ovine cerebral spinal fluid. Vet Immunol Immunopathol $124: 385-393$

[12] Mathivanan S, Fahner CJ, Reid GE, Simpson RJ (2012) ExoCarta (2012): database of exosomal proteins, RNA and lipids. Nucleic Acids Res. 40(Database issue):D1241-1244

[13] Connor DE, Exner T, Ma DD, and Joseph JE (2010) The majority of circulating platelet-derived microparticles fail to bind annexin $\mathrm{V}$, lack phospholipiddependent procoagulant activity and demonstrate greater expression of glycoprotein $\mathrm{Ib}$ Thromb Haemost 103:1044-1052

[14] Pant, S., Hilton, H. and Burczynski, M.E. (2012) The multifaceted exosome: biogenesis, role in normal and aberrant cellular function, and frontiers for pharmacological and biomarker opportunities. Biochem. Pharmacol., 83, 14841494.

[15] The'ry C, Ostrowski M, and Segura E (2009) Membrane vesicles as conveyors of immune responses. Nat Rev Immunol 9:581-593

[16] Lee Y, El Andaloussi S, Wood MJ (2012): Exosomes and microvesicles: extracellular vesicles for genetic information transfer and gene therapy. Hum Mol Genet. 21(R1):R125134

[17] Zakharova L, Svetlova M, Fomina AF (2007) T cell exosomes induce cholesterol accumulation in human monocytes via phosphatidylserine receptor. Journal of Cellular Physiology 212: 174-181

[18] Beyer C and Pisetsky DS (2010) The role of microparticles in the pathogenesis of rheumatic diseases. Nat Rev Rheumatol 6:21-29

[19] Beyer C and Pisetsky DS (2010) The role of microparticles in the pathogenesis of rheumatic diseases. Nat Rev Rheumatol 6:21-29

[20] Whitehead J, Pandey GK, Kanduri C (2009) Regulation of the mammalian epigenome by long noncoding RNAs. Biochim Biophys Acta, 1790:936-947.

[21] Giovanni Camussi and Peter J. Quesenberry (2013) Perspectives on the Potential Therapeutic Uses of Vesicles. Exosomes microvesicles, Vol. 1, 6

[22] Pegtel DM, van de Garde MD, and Middeldorp JM (2011) Viral miRNAs exploiting the endosomal-exosomal pathway for intercellular cross-talk and immune evasion. Biochim Biophys Acta 1809:715-721

[23] Giovanni Camussi, Maria-Chiara Deregibus, Stefania Bruno, Cristina Grange, Valentina Fonsato, Ciro Tetta (2011). Exosome/microvesicle-mediated epigenetic reprogramming of cells. Am J Cancer Res, 1(1): 98-110

[24] Ratajczak J, Wysoczynski M, Hayek F, Janowska- Wieczorek A, Ratajczak MZ (2006). Membrane- derived microvesicles: important and underappreciated mediators of cell-to-cell communication. Leukemia. 20:1487- 1495.

[25] Wimmer E, Mueller S, Tumpey TM, Taubenberger JK (2009).
Nature Biotechnology. 27(12):1163-72 Caspar DL, Klug A (1962). Physical principles in the construction of regular viruses. Cold Spring Harb. Symp. Quant. Biol.27:1-24

[26] Crick FH, Watson JD. Structure of small viruses (1956). Nature.177(4506):473-5.

[27] Manipulation of individual viruses: friction and mechanical properties (1997). Biophysical Journal. 72(3):1396-1403.

[28] Saunders, Venetia A.; Carter, John (2007). Virology: principles and applications. Chichester: John Wiley \& Sons; p. 72.

[29] Sandbulte MR, Westgeest KB, Gao J, Xu X, Klimov AI, Russell CA, Burke DF, Smith DJ, Fouchier RA, Eichelberger MC (2011). Proceedings of the National Academy of Sciences of the United States of America.108 (51):20748-53.

[30] Moss RB, Davey RT, Steigbigel RT, Fang F (2010).Targeting pandemic influenza: a primer on influenza antivirals and drug resistance. The Journal of Antimicrobial Chemotherapy.65 (6):1086-93.

[31] Hampson AW, Mackenzie JS. The influenza viruses (2006). Med. J. Aust.185(10 Suppl):S39-43.

[32] Lukashev AN (2005). Role of recombination in evolution of enteroviruses. Rev. Med. Virol.15 (3):157-67.

[33] Umene K (1999). Mechanism and application of genetic recombination in herpesviruses. Rev. Med. Virol.9 (3):171-82.

[34] Alwine JC (2008). Modulation of host cell stress responses by human cytomegalovirus. Curr. Top. Microbiol. Immunol.325:263-79.

[35] Margolis TP, Elfman FL, Leib D, et al (2007). Spontaneous reactivation of herpes simplex virus type 1 in latently infected murine sensory ganglia. J. Virol.81(20):11069-74

[36] Whitley RJ, Roizman B (2001). Herpes simplex virus infections. Lancet.357(9267):1513-8.

[37] Barton ES, White DW, Cathelyn JS, et al. (2007). Herpesvirus latency confers symbiotic protection from bacterial infection. Nature.447(7142):326-9.

[38] Bertoletti A, Gehring A (2007). Immune response and tolerance during chronic hepatitis B virus infection. Hepatol. Res.37 Suppl 3:S331-8.

[39] Akahata W, Yang ZY, Andersen H, et al. (March 2010). "A VLP vaccine for epidemic Chikungunya virus prtects nonhuman primates against infection" Nature Medicine 16 (3): $334-8$

[40] Goudsmit, Jaap (1998). Viral Sex. Oxford Univ Press.

[41] Barman S, Ali A, Hui EK, Adhikary L, Nayak DP (2001). Transport of viral proteins to the apical membranes and interaction of matrix protein with glycoproteins in the assembly of influenza viruses. Virus Res.77(1):61-9.

[42] Pegtel DM, van de Garde MD, and Middeldorp JM (2011) Viral miRNAs exploiting the endosomal-exosomal pathway for intercellular cross-talk and immune evasion. Biochim Biophys Acta 1809:715-721

[43] Nigel Dimmock, Andrew Easton, Keith Leppard (2006). Introduction to Modern Virology, 6th Edition. P71; WileyBlackwel. 
[44] Lee Y, El Andaloussi S, Wood MJ (2012) Exosomes and microvesicles: extracellular vesicles for genetic information transfer and gene therapy. Hum Mol Genet 21(R1):R125-134

[45] Eldh, M.; Ekström, K.; Valadi, H.; Sjöstrand, M.; Olsson, B.; Jernås, M.; Lötvall, J (2010) Exosomes communicate protective messages during oxidative stress; possible role of exosomal shuttle RNA. PLoS One, 5, 1-8.

[46] Montecalvo A, Larregina AT, Shufesky WJ, Stolz DB, Sullivan ML, Karlsson JM, Baty CJ, Gibson GA, Erdos G, Wang Z, et al. (2012) Mechanism of transfer of functional microRNAs between mouse dendritic cells via exosomes. Blood 119: 756-766.

[47] Bantel H, Schulze-Osthoff K (2003) Apoptosis in hepatitis C virus infection. Cell Death Differ; 10: S48-S58

[48] Lee SK, Park SO, Joe CO, Kim YS (2007) Interaction of HCV core protein with 14-3-3epsilon protein releases Bax to activate apoptosis. Biochem Biophys Res Commun; 352: 756-762

[49] Bartosch B, Cosset FL (2006) Cell entry of hepatitis C virus. Virology; 348: 1-12

[50] Vedashree Ramakrishnaiah, Christine Thumann, Isabel Fofana, Francois Habersetzer, Qiuwei Pan, Petra E. de Ruiter, Rob Willemsene, Jeroen A. A. Demmers, Victor Stalin Raj, Guido Jenster, Jaap Kwekkeboom, Hugo W. Tilanus, Bart L. Haagmans, Thomas F. Baumert, and Luc J. W. van der Laan. (2013). Exosome-mediated transmission of hepatitis C virus between human hepatoma Huh7.5 cells. PNAS Early Edition 1 of 5

[51] Soldaini E, et al. (2003) T cell costimulation by the hepatitis C virus envelope protein $\mathrm{E} 2$ binding to $\mathrm{CD} 81$ is mediated by Lck. Eur J Immunol 33(2):455-464.

[52] Masciopinto F, Giovani C, Campagnoli S et al (2004). Association of hepatitis $\mathrm{C}$ virus envelope proteins with exosomes. Eur J Immunol. 34: 2834-42

[53] Timpe JM, et al. (2008) Hepatitis C virus cell-cell transmission in hepatoma cells in the presence of neutralizing antibodies. Hepatology 47(1):17-24.

[54] Dreux M, et al. (2012) Short-range exosomal transfer of viral RNA from infected cells to plasmacytoid dendritic cells triggers innate immunity. Cell Host Microbe 12(4): 558-570.

[55] Bruggmann P, et al.; Swiss Hepatitis C Cohort Study (2008) Active intravenous drug use during chronic hepatitis C therapy does not reduce sustained virological response rates in adherent patients. J Viral Hepat 15(10):747-752.

[56] Fafi-Kremer S, et al. (2010) Viral entry and escape from antibody-mediated neutralization influence hepatitis $\mathrm{C}$ virus reinfection in liver transplantation. J Exp Med 207(9):20192031.

[57] Rashad S. Barsoum (2007) Hepatitis C virus: from entry to renal injury-facts and potentials Nephrol. Dial. Transplant.2 2 (7): $1840-1848$

[58] Lenassi M, et al. (2010) HIV Nef is secreted in exosomes and triggers apoptosis in bystander $\mathrm{CD} 4+\mathrm{T}$ cells. Traffic 11(1):110-122.

[59] Park IW and He JJ (2010) HIV-1 is budded from CD4 T lymphocytes independently of exosomes. Virol J 7:234
[60] Feng Z, et al. (2013) A pathogenic picornavirus acquires an envelope by hijacking cellular membranes. Nature 496(7445):367-371.

[61] Pegtel DM, Cosmopoulos K, Thorley-Lawson DA, van Eijndhoven MA, Hopmans ES, Lindenberg JL, de Gruijl TD, Wu" rdinger T, and Middeldorp JM (2010) Functional delivery of viral miRNAs via exosomes. Proc Natl Acad Sci USA 107:6328-6333.

[62] Meckes DG Jr, Shair KH, Marquitz AR, Kung CP, Edwards RH, and Raab-Traub N (2010) Human tumor virus utilizes exosomes for intercellular communication. Proc Natl Acad Sci USA 107:20370-20375

[63] Meckes DG Jr, Shair KH, Marquitz AR, Kung CP, Edwards RH, and Raab-Traub N (2010) Human tumor virus utilizes exosomes for intercellular communication. Proc Natl Acad Sci USA 107:20370-20375.

[64] Meckes DG Jr and Raab-Traub N (2011) Microvesicles and viral infection. J Virol 85:12844-12854

[65] Chiara Corrado, Stefania Raimondo, Antonio Chiesi, Francesco Ciccia, Giacomo De Leo, and Riccardo Alessandro (2013) Exosomes as Intercellular Signaling Organelles Involved in Health and Disease: Basic Science and Clinical Applications. Int. J. Mol. Sci. 14, 5338-5366

[66] Nelson, PN; Hooley, P and Molecular Immunology Research Group (October 2004)."Human endogenous retroviruses: Transposable elements with potential?".Clinical and Experimental Immunology 138 (138(1)): 1-9.

[67] Benjamin Kraus, Klaus Boller, Andreas Reuter and Barbara S Schnierle (2011). Characterization of the human endogenous retrovirus K Gag protein: identification of protease cleavage sites. Retrovirology, 8:21

[68] Dino Demirovic, Suresh I.S. Rattan (2013). Establishing cellular stress response profiles as biomarkers of homeodynamics, health and hormesis. Experimental Gerontology, 48; 94-98

[69] Feng Z, et al. (2013) A pathogenic picornavirus acquires an envelope by hijacking cellular membranes. Nature 496(7445):367-371.

[70] Deng ZB, Poliakov A, Hardy RW, Clements R, Liu C, Liu Y, Wang J, Xiang X, Zhang S, Zhuang X, et al. (2009) Adipose tissue exosome-like vesicles mediate activation of macrophage-induced insulin resistance. Diabetes 58:24982505

[71] Rautou PE, Leroyer AS, Ramkhelawon B, Devue C, Duflaut D, Vion AC, Nalbone G, Castier Y, Leseche G, Lehoux S, et al (2011) Microparticles from human atherosclerotic plaques promote endothelial ICAM-1-dependent monocyte adhesion and transendothelial migration. Circ Res 108:335-343

[72] Southcombe J, Tannetta D, Redman C, and Sargent I (2011) The immunomodulatory role of syncytiotrophoblast microvesicles. PLoS One 6:e20245.

[73] Boilard E, Nigrovic PA, Larabee K, Watts GF, Coblyn JS, Weinblatt ME, Massarotti EM, Remold-O'Donnell E, Farndale RW, Ware J, et al. (2010) Platelets amplify inflammation in arthritis via collagen-dependent microparticle production. Science 327:580-583 

Similarities between Extracellular Vesicles RNA (EVs RNA) and Chronic RNA Viruses

[74] Yong Zhao, Zhaoshun Jiang and Chengshan Guo (2011). New hope for type 2 diabetics: Targeting insulin resistance through the immune modulation of stem cells. Autoimmunity Reviews $11,137-142$.
[75] Feng D, Zhao WL, Ye YY, Bai XC, Liu RQ, Chang LF, et al(2010). Cellular internalization of exosomes occurs through phagocytosis. Traffic; 11: 675-687, doi: 10.1111/j.16000854.2010.01041.x.). 